\title{
Transmural Heterogeneity of the Action Potential Configuration in the Feline Left Ventricle
}

\author{
Takeshi Aiba, MD; Wataru Shimizu, MD*; Masashi Inagaki, MD; \\ Ichiro Hidaka, MS; Teiji Tatewaki, MD; Kenji Sunagawa, MD
}

\begin{abstract}
There are $\mathrm{M}$ cells in the canine, rabbit, guinea pig, and human left ventricle (LV), but it is not known if they are present in the feline LV. Arterially perfused feline LV preparations were used for the recording of transmembrane action potentials from the epicardium (Epi), midmyocardium (M) and endomyocardium (Endo) under control conditions $(\mathrm{n}=12)$ and in the presence of IKs blocker (chromanol 293B: 10u mol/L, $\mathrm{n}=6)$ or IKr blocker (E-4031: $2 \mu \mathrm{mol} / \mathrm{L}, \mathrm{n}=6$ ). The steady-state action potential duration at $90 \%$ repolarization and cycle length (APD90/CL) relation was obtained and fitted by the hyperbolic function $\mathrm{APD} 90=\mathrm{CL} /[(\mathrm{a} \times \mathrm{CL})+\mathrm{b}]$. In control, the shortest and longest action potential duration (APD) were observed in Epi and M, respectively, and the APD90/CL-relation curve was steeper in the M or Endo than in the Epi. Chromanol 293B prolonged APD in Epi, but not in M or Endo, resulting in no significant difference of the APD90/CL-relation curve among the 3 regions. E-4031 markedly, but homogeneously, prolonged APD in all regions, giving rise to decreased transmural dispersion of repolarization. In conclusion, there exists an M cell layer with a longer APD than the Epi and Endo layers and there is transmural electrical heterogeneity in the feline LV; however, the response to IKr blocker is different from that of the canine LV probably because of species differences in the IKr and IKs. (Circ J 2003; 67: 449-454)
\end{abstract}

Key Words: ECG; Ion channel; Membrane potential; Myocytes

$\mathbf{T}$ he regional differences in the electrophysiological properties of the ventricular myocardium have been well established!,2 Sicouri and Antzelevitch were the first to describe a subpopulation of cells (M cells) in the subepicardial and midmyocardial layers of the canine ventricular wall? They suggested that the M cell was distinguished electrophysiologically from other cells by the disproportionate prolongation of its action potential duration (APD) in response to slowing of the stimulation rate and to APD prolonging agents such as blockers of the rapidly activating delayed rectifier potassium current (IKr). The weaker, slowly activating delayed rectifier potassium current (IKs) and larger late sodium current $\left(\mathrm{INa}_{\mathrm{Na}}\right.$ in the $\mathrm{M}$ regions are reported to contribute to the longer APD in M cells than in other cell types4 Moreover, recent experiments using wedge preparations have shown that the electrophysiological heterogeneity across the ventricular wall produced the inscription of the normal and abnormal $\mathrm{T}$ wave and was responsible for the occurrence of ventricular tachyarrhythmias ${ }^{5-9}$

The presence of $\mathrm{M}$ cells has been shown in the deep layers of the canine, pig, rabbit, guinea pig, and human left ventricle (LV), ${ }^{3,10-13}$ The feline heart is commonly used in experimental studies examining the effect of myocardial ischemia ${ }^{14-17}$ ventricular hypertrophy, ${ }^{18,19}$ and sympathetic and parasympathetic nerve activity 20,21 on ventricular

(Received December 18, 2002; revised manuscript received February 4, 2003; accepted February 25, 2003)

Department of Cardiovascular Dynamics, Research Institute and *Division of Cardiology, Department of Internal Medicine, National Cardiovascular Center, Suita, Japan

Mailing address: Wataru Shimizu, MD, PhD, Division of Cardiology, Department of Internal Medicine, National Cardiovascular Center, 5-7-1 Fujishiro-dai, Suita 565-8565, Japan. E-mail: wshimizu@ @sp. ncvc.go.jp action potentials. Although previous studies by Myerburg et al showed the ionic and cellular basis for the electrophysiological difference between epicardial and endocardial cells in the feline ventricle ${ }^{22-24}$ its transmural heterogeneity of APD and the presence of M cell remains unknown. The purpose of this investigation was to assess the electrophysiological and pharmacological characteristics of epicardial, midmyocardial, and endocardial cells in the feline LV. The results indicated that transmural electrophysiological heterogeneity existed in the feline LV.

\section{Methods}

Arterially Perfused Wedge of Feline Ventricle

Cats weighting $2.0-4.0 \mathrm{~kg}$ were anesthetized with intraperitoneal pentobarbital $(1.0 \mathrm{mg} / \mathrm{kg})$. The chest was opened via a left thoracotomy, and the heart was excised and placed in a cardioplegic solution consisting of cold $\left(4^{\circ} \mathrm{C}\right)$ Tyrode's solution containing $8.5 \mathrm{mmol} / \mathrm{L}\left[\mathrm{K}^{+}\right]$and transmural wedges with dimensions of approximately $2 \times 1 \times$ $0.5 \mathrm{~cm}$ to $3 \times 1 \times 1 \mathrm{~cm}$ were dissected from the anterior wall of the LV. The tissue was cannulated via a branch of the left descending coronary artery with cardioplegic solution. The preparation was then placed in a small tissue bath and arterially perfused by a roller pump (Cole Parmer Instrument Co) with Tyrode's solution of the following composition (mmol/L): $129 \mathrm{NaCl}, 4 \mathrm{KCl}, 0.9 \mathrm{NaH}_{2} \mathrm{PO} 4,20 \mathrm{NaHCO}_{3}$, $1.8 \mathrm{CaCl}_{2}, 0.5 \mathrm{MgSO}_{4}$, and 5.5 glucose, buffered with $95 \%$ $\mathrm{O}_{2}$ and $5 \% \mathrm{CO}_{2}\left(37 \pm 1^{\circ} \mathrm{C}\right)$. Perfusion pressure was maintained between 40 and $60 \mathrm{mmHg}$ by adjusting the flow rate of the pump. The ventricular wedges were allowed to equilibrate in the tissue bath until electrically stable for $1 \mathrm{~h}$ and stimulated with bipolar electrodes applied to the endocardial surface. 


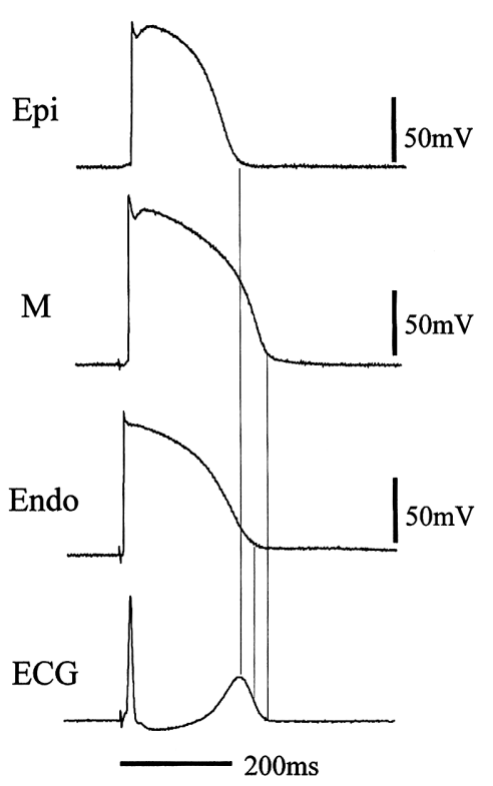

Fig 1. Transmembrane action potentials (AP) and a transmural ECG simultaneously recorded from a feline left ventricular wedge preparation under baseline conditions. The AP were recorded from the epicardial (Epi), longest midmyocardial (M), and endomyocardial (Endo) regions at a basic cycle length of $1,000 \mathrm{~ms}$ using floating glass microelectrodes. The briefest AP in the Epi coincided with the peak of the T wave in the ECG, whereas the longest action potential in the $M$ region coincided with the end of the $T$ wave.

\section{Recording of ECG and Transmembrane Action Potentials (AP)}

A transmural ECG was recorded with $\mathrm{Ag}-\mathrm{AgCl}$ electrodes, which were placed in the Tyrode's solution bathing the preparation, $1.0 \mathrm{~cm}$ from the epicardial and endocardial surfaces (epicardial, positive pole). Transmembrane AP in the wedge preparations were recorded simultaneously from the epicardium, midmyocardium, and endomyocardium by the use of 3 separate intracellular floating microelectrodes (DC resistance $10-20 \mathrm{M} \Omega ; 2.7 \mathrm{mmol} / \mathrm{L} \mathrm{KCl}$ ) connected to a high-input impedance amplification system (World Precision Instruments). We recorded epicardial and endocardial AP from the epicardial and endocardial surfaces of the preparations at positions approximating the transmural axis of the ECG recording; the midmyocardial AP were recorded at the area between the epicardium and endocardium along the same axis. All amplified signals were digitized at $2 \mathrm{kHz}$ using a 12-bit analog-to-digital converter and stored on a hard disk of a dedicated laboratory computer system, and analyzed using the original software of our laboratory. All APD measurements were reported at $90 \%$ repolarization (APD90). Transmural dispersion of repolarization (TDR) was defined as the difference between the longest and shortest repolarization times (activation time+ APD90) of AP recorded across the wall (M-epicardial region). The recordings of ECG and transmembrane AP in the feline LV were similar to those reported using wedge preparations ${ }^{5-9}$

\section{Study Protocol}

Transmural ECG and transmembrane AP were recorded in the absence (control condition) and presence of the IKs blocker (chromanol 293B: 10u mol/L) or IKr blocker (E4031: $2 \mu \mathrm{mol} / \mathrm{L}$ ). Then, we studied the relation between APD90 and cycle length (CL) at steady state pacing of

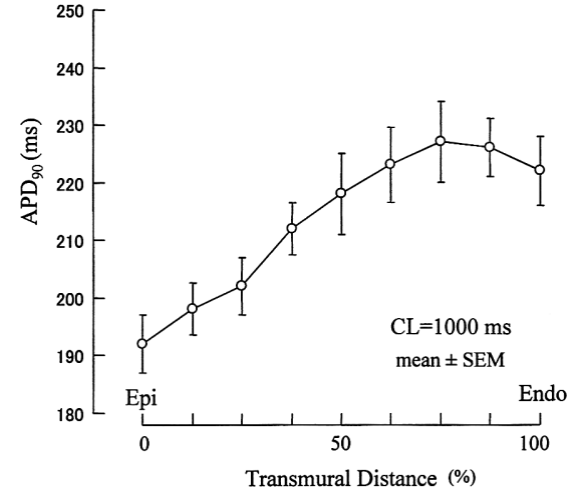

Fig 2. Distribution of the action potential duration at $90 \%$ repolarization (APD90) across the feline left ventricular wall at a cycle length (CL) of $1,000 \mathrm{~ms}$ under control conditions. Transmural distance at $0 \%$ and $100 \%$ represent the epicardium (Epi) and endomyocardium (Endo), respectively. Midmyocardial action potentials were recorded at the area between the epicardium and endocardium along the same axis. Data represent mean $\pm \operatorname{SEM}(\mathrm{n}=5)$.

$2,000,1,500,1,000$, and $500 \mathrm{~ms}$.

\section{Data Analysis}

APD90 measured at steady state was plotted as a function of pacing CL and these experimental points were accurately fitted ( $r>0.95)$ by the hyperbolic relation introduced for this purpose by Elharrar et al: 25,26

$$
\mathrm{APD} 90=\mathrm{CL} /[(\mathrm{a} \times \mathrm{CL})+\mathrm{b}]
$$

In this equation, the coefficients ' $a$ ' and ' $b$ ' define the degree of APD90 dependence on CL. The term 1/a represents the asymptotic value of APD 90 at an infinitely long CL $(1 / a=A P D \max )$; that is, the one that would occur if an AP was stimulated in a quiescent fiber. Thus, APD max is independent of rate, and is thought to reflect the intrinsic membrane properties that determine the time course of repolarization. The $\mathrm{CL}$ at which $\mathrm{APD}$ equals $50 \%$ of APDmax (CL50) can be estimated from ' $b$ ' and APDmax as follows:

$$
\mathrm{CL} 50=\mathrm{b} \times \mathrm{APDmax}
$$

\section{Statistical Analysis}

Estimation of the parameters describing the APD90/CL relation at steady state was performed by a linear least square fitting between 1/APD 90 and 1/CL. The goodness of fit was tested by the correlation coefficient $r>0.95$. Mean values of the variables among groups were tested using ANOVA. Individual post hoc comparisons were made using Bonferroni/Dunn test. Data are expressed as mean \pm SD values, except for those shown in the Figs, which are expressed as mean \pm SEM values. Significance was defined as a value of $\mathrm{p}<0.05$.

\section{Results}

\section{AP Configuration and Distribution}

Fig 1 illustrates a representative transmembrane AP simultaneously recorded from the epicardium, the longest midmyocardium ( $\mathrm{M}$ region), and the endocardium together with a transmural ECG at the CL of $1,000 \mathrm{~ms}$ under control conditions. The AP of the epicardium and $\mathrm{M}$ region typically exhibited a distinct spike and dome morphology, 




Fig 3. Transmembrane action potentials (AP) simultaneously recorded from the epicardial (Epi), longest midmyocardial (M), and endomyocardial (Endo) regions across the feline left ventricular wall at cycle lengths (CL) of 500,1,000, and 2,000 ms under baseline (con-


B), and E-4031 ( $2 \mu \mathrm{mol} / \mathrm{L})$ (Panel C). Chromanol 293B slightly prolonged the action potential duration (APD) in the epicardial and endocardial regions only, not in the $\mathrm{M}$ region. E-4031 pronouncedly prolonged the APD in all regions.

whereas those of the endocardial cell lacked the dome and exhibited a negatively sloping phase-2. In comparison with the transmural ECG, repolarization of the briefest AP in the epicardium coincided with the peak of the $\mathrm{T}$ wave, whereas the longest AP in the M region coincided with the end of the $\mathrm{T}$ wave. The repolarization of the endocardium was intermediate between that of the epicardium and M region. Fig 2 shows the composite data $(\mathrm{n}=5)$ of the transmural APD distribution at a CL of $1,000 \mathrm{~ms}$ across the feline $\mathrm{LV}$ in control conditions. The APD90 was briefest at the epicardium, gradually increased, and became longest at the subendocardium. These transmembrane AP configurations and the transmural APD distribution in our experiments were similar to those in canine ventricular wedge preparations ${ }^{5-9,19}$

\section{Effects of IKs or IKr Blocker on APD/CL Relationship}

Fig 3 illustrates a superimposed transmembrane AP recorded from the epicardial, $\mathrm{M}$, and endocardial regions at CLs of 500, 1,000, and 2,000 ms (steady-state condition) in the control state and after chromanol 293B (10u mol/L) and E-4031 ( $2 \mu \mathrm{mol} / \mathrm{L})$. Slowing the stimulation rate prolonged the APD in the 3 regions in the control condition, after
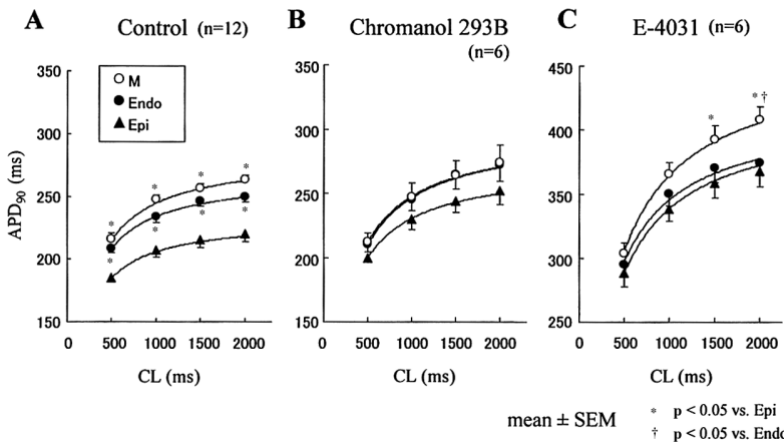

Fig 4. Composite data of the relation between action potential duration measured at $90 \%$ repolarization (APD90) and pacing cycle length (CL) in the epicardial (Epi), midmyocardial (M), and endomyocardial (Endo) regions of the feline left ventricular wall under baseline (control) condition ( $\mathrm{n}=12)$ (Panel A), after chromanol 293B (n=6) (Panel B), and E-4031 $(n=6)$ (Panel C). Continuous curves were computed from the hyperbolic fitting method. Data are mean \pm SEM. $* \mathrm{p}<0.05$ vs Epi, ${ }^{\dagger} \mathrm{p}<0.05$ vs Endo.

chromanol 293B and after E-4031.

Fig 4 and Table 1 show the composite data of the APD90/CL relationship in the control state $(\mathrm{n}=12)$, and after chromanol 293B $(n=6)$ and E-4031 $(n=6)$. In control conditions, the APDmax was significantly larger in the $\mathrm{M}$ and endocardial regions than in the epicardium $(p<0.01)$, but CL50 was not significantly different among the 3 regions (Table 1). Therefore, the APD90/CL-relation curve was steeper in the $\mathrm{M}$ or endocardial region than in the epicardium under control conditions (Fig 4, Panel A). Chromanol 293B (10u mol/L) increased the APDmax in the epicardium $(\mathrm{p}<0.05)$, but not in the $\mathrm{M}$ or endocardial region, resulting in no significant difference in APDmax among the 3 regions. Moreover, the CL50 was not significantly different among the 3 regions after chromanol 293B. Therefore, chromanol 293B caused the APD90/CL-relation curve homogeneously in the 3 regions (Fig 4, Panel B). On the other hand, E-4031 $(2 \mu \mathrm{mol} / \mathrm{L})$ markedly increased the APDmax in all 3 regions, and it was larger in the $\mathrm{M}$ region than in the epicardium or endocardium $(\mathrm{p}<0.05)$. The CL50 was also prolonged after E-4031 ( $<<0.01)$, but was not significantly different among the 3 regions. Therefore, E-4031 steepened the APD90/CLrelation curve, especially in the M region (Fig 4, Panel C).

Effects of IKs or IKr Blockers on TDR

Fig 5 illustrates a superimposed transmembrane AP

Table 1 Parametric Description on the Steady-State APD90/CL Relation

\begin{tabular}{|c|c|c|c|c|c|}
\hline & Region & $a\left(\times 10^{-3}\right)\left(\mathrm{ms}^{-1}\right)$ & $b^{\#}$ & $A P D_{\max }(m s)$ & $C L 50(\mathrm{~ms})$ \\
\hline \multirow{3}{*}{$\begin{array}{l}\text { Control } \\
(n=12)\end{array}$} & Epi & $4.2 \pm 0.37$ & $0.58 \pm 0.09$ & $239 \pm 22$ & $140 \pm 30$ \\
\hline & $M$ & $3.5 \pm 0.15 *$ & $0.57 \pm 0.17$ & $285 \pm 13 *$ & $162 \pm 49$ \\
\hline & Endo & $3.7 \pm 0.28 *$ & $0.54 \pm 0.13$ & $269 \pm 18 *$ & $145 \pm 37$ \\
\hline \multirow{3}{*}{$\begin{array}{l}\text { Chromanol } \\
293 B \\
(n=6)\end{array}$} & Epi & $3.7 \pm 0.49^{\dagger}$ & $0.67 \pm 0.22$ & $277 \pm 43^{\dagger}$ & $194 \pm 96$ \\
\hline & $M$ & $3.4 \pm 0.43$ & $0.68 \pm 0.11$ & $302 \pm 44$ & $209 \pm 64$ \\
\hline & Endo & $3.5 \pm 0.45$ & $0.68 \pm 0.14$ & $289 \pm 43$ & $201 \pm 76$ \\
\hline \multirow{3}{*}{$\begin{array}{l}E-4031 \\
(n=6)\end{array}$} & Epi & $2.5 \pm 0.19^{\ddagger}$ & $0.51 \pm 0.11$ &  & $207 \pm 49^{\dagger}$ \\
\hline & $M$ & $2.2 \pm 0.16^{\ddagger *}$ & $0.56 \pm 0.08$ & $461 \pm 34^{+*}$ & $260 \pm 41^{*}$ \\
\hline & Endo &  & $0.50 \pm 0.17$ & $420 \pm 39^{+}$ & $213 \pm 88$ \\
\hline
\end{tabular}

Epi, epicardium; M, midmyocardium; Endo, endomyocardium. ${ }^{\# D i m e n s i o n l e s s . ~}{ }^{*} p<0.05$ vs Epi, ${ }^{\dagger} p<0.05$ vs control, ${ }^{\ddagger} p<0.01$ vs control. 
A

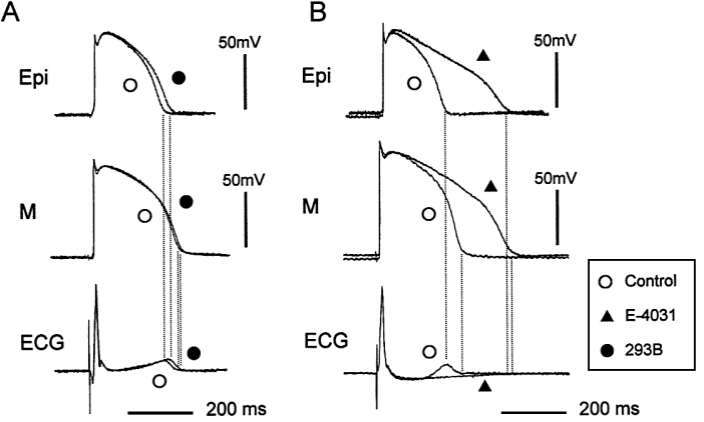

Fig 5. Superimposed transmembrane action potentials recorded from the epicardial (Epi) and $\mathrm{M}$ regions, and the transmural ECG at cycle length of $1,000 \mathrm{~ms}$ before (control) and after chromanol 293B (Panel A) and E-4031 (Panel B).

recorded from the epicardial and $\mathrm{M}$ regions and transmural ECG at BCL of 1,000 ms before and after chromanol 293B (Fig 5, Panel A) and E-4031 (Fig 5, Panel B). Chromanol 293B slightly prolonged the APD in the epicardium, but not in the $\mathrm{M}$ region, resulting in decreasing TDR without any change of QT interval. On the other hand, E-4031 prolonged APD markedly, but homogeneously, in the epicardial and $\mathrm{M}$ regions, resulting in increasing QT interval, but decreasing TDR. Fig 6 shows the composite data of the APD $90 / C L$ relationship in the epicardial, $\mathrm{M}$, and endocardial regions and TDR before and after chromanol 293B or E-4031. Chromanol 293B slightly prolonged the APD90 in the epicardium (Fig 6, Panel A), but not in the $\mathrm{M}$ and endocardial regions (Fig 6, Panels B and C), resulting in decreasing TDR (Fig 6, Panel D). On the other hand, E-4031 markedly and homogeneously prolonged APD90 in the 3 regions (Fig6, Panels A-C), and decreased TDR (Fig6, Panel D).

\section{Discussion}

We have shown that the electrophysiological characteristics and pharmacological responsiveness were transmurally heterogeneous in the perfused feline LV. We found a subpopulation of cells in the midmyocardium with a longer APD, moderate phase-1 notch and steeper APD/CL-relation curve under control conditions and in the presence of an IKr blocking agent. These characteristics are similar to those of $\mathrm{M}$ cells previously reported in other mammalian species, including humans, thereby suggesting transmural electrical heterogeneity in the feline LV.

\section{Transmural AP Heterogeneity}

The regional difference in AP configuration between the epicardial, M, and endocardial cells has been well demonstrated in canine ventricular wedge, tissue and myocyte preparations ${ }^{3-9,27-29}$ In the feline LV, several experiments have demonstrated the characteristics and differences in AP between the epicardium and endocardium, 14,15,22,23 but not of the midmyocardium. The present study is, to our best knowledge, the first to show transmural heterogeneity of the electrophysiological characteristics and pharmacological responses in the intact feline LV. The shortest and longest APD in the feline LV were observed in the epicardium and subendocardium (M region), respectively, consistent with those in the canine wedge preparation ${ }^{5-9,19}$ Moreover, the AP in the feline epicardium and $\mathrm{M}$ region had a
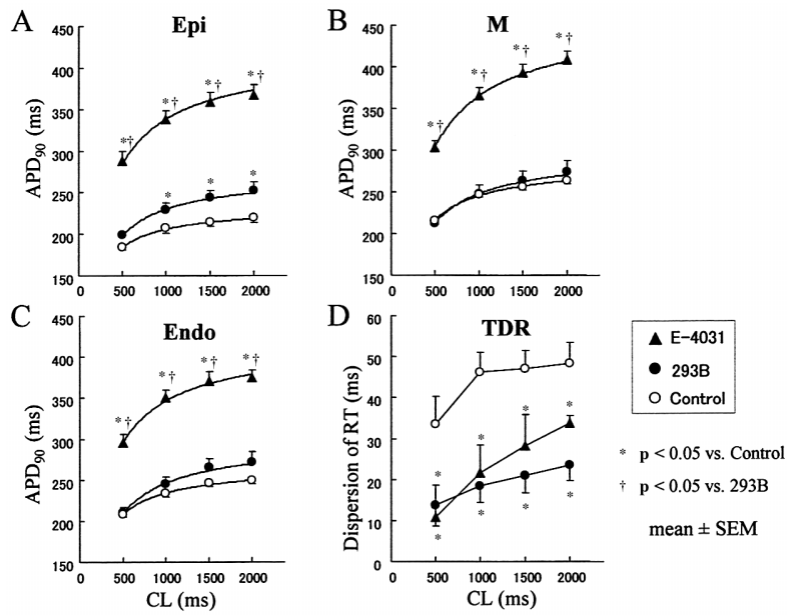

Fig 6. Composite data of the relation between action potential duration measured at $90 \%$ repolarization (APD90) and cycle length (CL) under baseline (control) conditions $(\mathrm{n}=12)$, after chromanol 293B $(n=6)$ and after E-4031 $(n=6)$ in the epicardial (Epi) (Panel A), midmyocardial (M) (Panel B), and endomyocardial (Endo) (Panel C) regions, and the transmural dispersion of repolarization (TDR) (Panel D) across the feline left ventricular wall. Continuous curves in Panels A-C were computed from the hyperbolic fitting method. Data are mean \pm SEM. ${ }^{*} \mathrm{p}<0.05$ vs control, ${ }^{\dagger} \mathrm{p}<0.05$ vs chromanol $293 \mathrm{~B}$.

phase- 1 notch, whereas that in the endocardium lacked the notch and exhibited a negatively sloping phase-2. Furthermore, the correlations between the AP and transmural ECG in the feline wedge were similar to those in previous canine experiments. These findings suggest that the feline ventricular AP configuration and distribution across the transmural surface are similar to those in the canine ventricle. There exists the M-cell like layer in the feline LV, but its behavior is not identical to other animal species.

\section{Characteristics of M Cells in the Feline LV}

Marked AP prolongation with slowing of the stimulation rate is one of the characteristics of the $\mathrm{M}$ cell in the canine LV!-3 The APD90 in the M region is longer than that in the epicardium and endocardium, but the APD90/CL-relation curve under control conditions in the present study was not so much steeper in the $\mathrm{M}$ region than in the epicardium or endocardium. It is conceivable that intact cell-to-cell coupling in the wedge preparation might cause the APD90/CLrelation curve to be flatter in the $\mathrm{M}$ region compared with isolated tissue preparations and myocytes? ${ }^{27}$

Another characteristic of the feline $\mathrm{M}$ cell is its unique sensitivity to IKr blocking agents. In the previous canine wedge experiments, IKr block with d-sotalol produced greater prolongation of the APD in the M cell than in the epicardial and endocardial cells, resulting in an increase of TDR5 In the present feline LV preparation, the APD90/CLrelation curve became much steeper in all regions after IKr block with E-4031, resulting in a homogeneously prolonged APD and decreased TDR, which is in striking contrast to the results in canine wedge preparations. The reason for this difference is the differential distribution and ratio of IKr and IKs across the feline LV.

\section{Ionic Currents}

In the canine ventricle, the IKr current is homogeneous across the myocardial layers, but the IKs current was weaker in the $\mathrm{M}$ region than in the epicardium and endocardium, 
contributing to the longer APD in the M cells. In contrast, the distribution of IKr and IKs across the feline LV has never been demonstrated. Chapula reported the existence of 2 components of the delayed rectifier $\mathrm{K}^{+}$current (IKr and IKs) in cat ventricular myocytes ${ }^{30}$ Follmer et al reported that IKr blockade by flecainide and E-4031 almost completely reduced the delayed rectifier $\mathrm{K}^{+}$current (IK) ${ }^{24}$ although they did not show which cell type from the transmural layers was examined. Moreover, Furukawa et al showed that total IK was greater in the epicardium than in the endocardium in the feline ventricle, and that the regional IK difference contributed to the shorter APD in the epicardium than in the endocardium ${ }^{22}$ These findings suggest that the IK in the feline LV is mainly dependent on IKr, which is consistent with our data showing a prominent prolongation of APD90 in all 3 layers with E-4031, but only a slight prolongation of APD with chromanol 293B.

Moreover, IKs block with chromanol 293B preferentially prolonged APD in the epicardium (206 \pm 23 to $230 \pm 20 \mathrm{~ms}$ ) rather than in the $M$ region $(247 \pm 28$ to $247 \pm 26 \mathrm{~ms})$ and endocardium ( $234 \pm 15$ to $245 \pm 21 \mathrm{~ms}$ ) at the CL of $1,000 \mathrm{~ms}$. IKr block with E-4031 also preferentially prolonged APD in the epicardium (206 \pm 23 to $343 \pm 26 \mathrm{~ms}$ ) rather than in the M region $(252 \pm 28$ to $366 \pm 24 \mathrm{~ms})$ and endocardium $(234 \pm 15$ to $351 \pm 24 \mathrm{~ms}$ ) at the CL of $1,000 \mathrm{~ms}$. These findings suggested that both IKr and IKs are larger in the epicardial cells than in the $\mathrm{M}$ and endocardial cells in the feline heart. Recently, we showed that IKr block based on IKs block markedly prolonged the QT interval and TDR, and induced early afterdepolarizations and torsades de pointes in the feline LV wedge preparation. 31 Therefore, we suggest that these characteristics of the feline heart (IKr dependent) can be used to mimic drug-induced QT prolongation and torsades de pointes.

\section{Study Limitations}

The effects of the IKr and IKs blockers on the prolongation of APD in each region suggested a regional difference in IKr and IKs densities, but we did not directly record the $\mathrm{IKr}$ and IKs density of isolated feline ventricular myocytes in each region. It is difficult to explain all the characteristics and behaviors of the AP in the feline LV using only the IKr and Iks. The contribution of other currents (especially INa, ICa,L, Ito, IK1) needs to be taken into account.

Our data showed a longer APD in the M region after E4031, but we have never observed early afterdepolarization and torsades de pointes in this preparation, which might be because (1) we only paced at CL of 500-2,000 ms, and (2) E-4031 markedly, but homogenously, prolonged APD, resulting in reducing TDR.

\section{Acknowledgments}

We are grateful to Masahiko Kondo for technical assistance and helpful suggestions. Chromanol 293B was generously donated by Hoechst Marion Roussel.

Dr Shimizu was supported in part by the Japanese Cardiovascular Research Foundation, Vehicle Racing Commemorative Foundation, and Health Sciences Research Grants from the Ministry of Health, Labour and Welfare, Japan.

\section{References}

1. Antzelevitch C, Sicouri S, Lukas A, Nesterenko VV, Liu DW, Di Diego JM. Regional differences in the electrophysiology of ventricular cells: Physiological and clinical implications. In: Zipes DP, Jalife J, editors. Cardiac electrophysiology: From cell to bedside. Philadelphia: WB Saunders; 1995: 228-245.
2. Antzelevitch C, Shimizu W, Yan GX, Sicouri S, Weissenburger J, Nesterenko VV, et al. The M cell: Its contribution to the ECG and to normal and abnormal electrical function of the heart. $J$ Cardiovasc Electrophysiol 1999; 10: 1124-1152.

3. Sicouri S, Antzelevitch C. A subpopulation of cells with unique electrophysiological properties in the deep subepicardium of the canine ventricle: The presence of M cell. Circ Res 1991; 68: 1729-1741.

4. Liu DW, Antzelevitch C. Characteristics of the delayed rectifier current (IKr and IKs) in canine ventricular epicardial, midmyocardial, and endocardial myocytes: A weaker IKs contributes to the longer action potential of the M cell. Circ Res 1995; 76: 351-365.

5. Shimizu W, Antzelevitch C. Sodium channel block with mexiletine is effective in reducing dispersion of repolarization and preventing torsade de pointes in LQT2 and LQT3 models of the long-QT syndrome. Circulation 1997; 96: 2038-2047.

6. Yan GX, Antzelevitch C. Cellular basis for the normal T wave and the electrocardiographic manifestations of the long-QT syndrome. Circulation 1998; 98: 1928-1936.

7. Shimizu W, Antzelevitch C. Cellular basis for the electrocardiographic features of the LQT1 form of the long QT syndrome: Effects of $\beta$-adrenergic agonists, antagonists, and sodium channel block on transmural dispersion of repolarization and torsade de pointes. Circulation 1998; 98: 2314-2322.

8. Shimizu W, Antzelevitch C. Cellular and ionic basis for T wave alternans under long QT conditions. Circulation 1999; 99: 1499_ 1507.

9. Yan GX, Wu Y, Liu T, Wang J, Marinchak RA, Kowey PR. Phase 2 early afterdepolarization as a trigger of polymorphic ventricular tachycardia in acquired long-QT syndrome: Direct evidence from intracellular recording in the intact left ventricular wall. Circulation 2001; 103: 2851-2856.

10. Sicouri S, Quist M, Antzelevitch C. Evidence for the presence of M cells in the guinea pig ventricle. J Cardiovasc Electrophysiol 1996; 7: $503-511$.

11. Weirich J, Bernhardt R, Loewen N, Wenzel W, Antoni H. Regionaland species-dependent effects of $\mathrm{K}^{+}$-channel blocking agents on subendocardium and mid-wall slices of human, rabbit, and guinea pig myocardium (abstract). Pflugers Arch 1996; 431: R130.

12. Stankovicova T, Szilard M, Scheerder ID, Sipido KR. M cells and transmural heterogeneity of action potential configuration in myocytes from the left ventricular wall of the pig heart. Cardiovasc Res 2000; 45: 952-960.

13. Drouin E, Charpentier F, Gautheier C, Laurent K, Marec HL. Electrophysiologic characteristics of cells spanning the left ventricular wall of human heart: Evidence for presence of M cells. $J$ Am Coll Cardiol 1995; 26: 185-192.

14. Kimura S, Bassett AL, Kohya T, Kozlovskis PL, Myerburg RJ. Simultaneous recording of action potentials from endocardium and epicardium during ischemia in the isolated cat ventricle: Relation of temporal electrophysiologic heterogeneities to arrhythmias. Circulation 1986; 74: 401-409.

15. Kimura S, Bassett AL, Furukawa T, Cuevas J, Myerburg RJ. Electrophysiological properties and responses to simulated ischemia in cat ventricular myocytes of endocardial and epicardial origin. Circ Res 1990; 66: 469-477.

16. Kimura S, Bassett AL, Cameron JS, Huikuri H, Kozlovskis PL, Myerburg RJ. Cellular electrophysiological changes during ischemia in isolated, coronary-perfused cat ventricle with healed myocardial infarction. Circulation 1988; 78: 401-406.

17. Furukawa T, Kimura S, Furukawa N, Bassett AL, Myerburg RJ. Role of ATP-regulated potassium channels in differential responses of endocardial and epicardial cells to ischemia. Circ Res 1991; 68: $1693-1702$.

18. Furukawa T, Myerburg RJ, Furukawa N, Kimura S, Bassett AL. Metabolic inhibition of ICa, L and Ik differs in feline left ventricular hypertrophy. Am J Physiol 1994; 266: H1121 - H1131.

19. Kleiman RB, Houser S. Outward currents in normal and hypertrophied feline ventricular myocytes. Am J Physiol 1989; 256: H1450H1461.

20. Zaza A, Malfatto G, Schwartz PJ. Sympathetic modulation of the relation between ventricular repolarization and cycle length. Circ Res 1991; 68: 1191-1203.

21. Malfatto G, Zaza A, Schwartz PJ. Parasympathetic control of cycle length dependence of endocardial ventricular repolarization in the intact feline heart during steady state conditions. Cardiovasc Res 1993; 27: 823-827.

22. Furukawa T, Kimura S, Furukawa N, Bassett AL, Myerburg RJ. Potassium rectifier currents differ in myocytes of endocardial and epicardial origin. Circulation 1992; 70: 91-103.

23. Furukawa T, Myerburg RJ, Furukawa N, Bassett AL, Kimura S. 
Differences in transient outward currents of feline endocardial and epicardial myocytes. Circ Res 1990; 67: 1287-1291.

24. Follmer $\mathrm{CH}$, Colatsky TJ. Block of delayed rectifier potassium current, IK, by flecainide and E-4031 in cat ventricular myocytes. Circulation 1990; 82: 289-293.

25. Elharrar V, Surawicz B. Cycle length effect on restitution of action potential duration in dog cardiac fibers. Am J Physiol 1983; 244: $\mathrm{H} 782-\mathrm{H} 792$

26. Elharrar V, Atarashi H, Suravicz B. Cycle length-dependent action potential duration in canine cardiac Purkinje fibers. Am J Physiol 1984; 247: H936-H945.

27. Yan GX, Shimizu W, Antzelevitch C. Characteristics and distribution of $\mathrm{M}$ cells in arterially perfused canine ventricular wedge preparations. Circulation 1998; 98: 1921-1927.
28. Sicouri S, Fish J, Antzelevitch C. Distribution of $\mathrm{M}$ cells in the canine ventricle. J Cardiovasc Electrophysiol 1994; 5: 824-837.

29. Burashnikov A, Antzelevitch C. Prominent IKs in epicardium and endocardium contributes to development of transmural dispersion of repolarization but protects against development of early afterdepolarizations. J Cardiovasc Electrophysiol 2002; 13: 172-177.

30. Chapula JS. Increase in action potential duration and inhibition of the delayed rectifier outward current Ік by berberine in cat ventricular myocytes. Br J Pharmacol 1996; 117: 1427-1434.

31. Aiba T, Shimizu W, Hidaka I, Uemura K, Sunagawa K. Verapamil suppresses torsade de pointes by reducing transmural dispersion of repolarization and preventing early afterdepolarization in acquired model of long-QT syndrome. Circulation 2002; 106(Suppl): II-22. 